\title{
Short-term variability of Sgr A*
}

\author{
M. R. Morris ${ }^{1}$ S. D. Hornstein, ${ }^{1}$ A. M. Ghez,${ }^{1}$ J. R. Lu, ${ }^{1}$ \\ K. Matthews ${ }^{2}$ and F. K. Baganoff ${ }^{3}$ \\ ${ }^{1}$ Dept. of Physics \& Astronomy, Univ. of California, Los Angeles, CA 90095-1547, USA \\ ${ }^{2}$ Caltech Optical Observatories, California Institute of Technology, Mail Code 105-24, \\ Pasadena, CA 91125, USA \\ ${ }^{3}$ Center for Space Research, Massachusetts Institute of Technology, \\ Cambridge, MA 02139, USA \\ email: morris@astro.ucla.edu
}

\begin{abstract}
Observations of Sgr A* over the past 4 years with the Keck Telescope in the nearinfrared, coupled with millimeter and submillimeter observations, show that the $3.7 \times 10^{6} M_{\odot}$ Galactic Black Hole, Sagittarius A*, displays continuous variability at all these wavelengths, with the variability power concentrated on characteristic time scales of a few hours, and with a variability fraction that increases with wavelength. We review the observations indicating that the few-hour time scale for variability is reproduced at all accessible wavelengths. Interpreted as a dynamical time, this time scale corresponds to a radial distance of $2 \mathrm{AU}$, or $\sim 25$ Schwarzschild radii. Searches for quasi-periodicities in the near-infrared data from the Keck Telescope have so far been negative. One interpretation of the character of these variations is that they result from a recurring disk instability, rather than from variations in the mass accretion rate flowing through the outer boundary of the emission region. However, neither a variable accretion rate nor a mechanism associated with a jet can presently be ruled out.
\end{abstract}

Keywords. Galaxy: nucleus - black hole physics - accretion disks

\section{Radio variability}

The Galactic black hole, Sgr A*, has been observed across the electromagnetic spectrum from radio to X-rays, except where interstellar dust blocks our view. Furthermore, it is variable at all accessible wavelengths. It has been known for some time that this source is variable in the radio on time scales from weeks to years (Brown \& Lo 1982; Zhao et al. 1989, 1992; Herrnstein et al. 2004). The variability at the longer centimeter wavelengths is ascribable to interstellar scintillation, but at a few $\mathrm{cm}$ and shorter wavelengths, the variations are largely intrinsic (e.g., Macquart \& Bower 2006). The amplitude of the intensity modulation depends on the time scale, but there is a general trend for the amplitude to decrease with increasing wavelength (Herrnstein et al. 2004). Relatively weak variability $(\sim 10 \%)$ of the 1.3 and 0.7 -cm emission from Sgr A* on an hourly time scale (Yusef-Zadeh et al. 2006) is consistent with that trend; this result was interpreted in terms of an expanding plasmon model because of a half-hour time lag between the peaks of a flare at these two frequencies.

Variability on hourly time scales is particularly interesting, as such time scales correspond to light travel times across distances of $<100$ Schwarzschild radii $\left(R_{s}\right)$. Intraday variability of 3-mm emission was reported by Miyazaki et al. (2004, 2006). They noted "flares" occurring on time scales of a few weeks to a month that have maximum amplitude increases of $100 \div 200 \%$ at $100 \mathrm{GHz}$ and $200 \div 400 \%$ at $140 \mathrm{GHz}$, and on a number of occasions they noted significant factor-of-two variability on time scales of a few hours. 


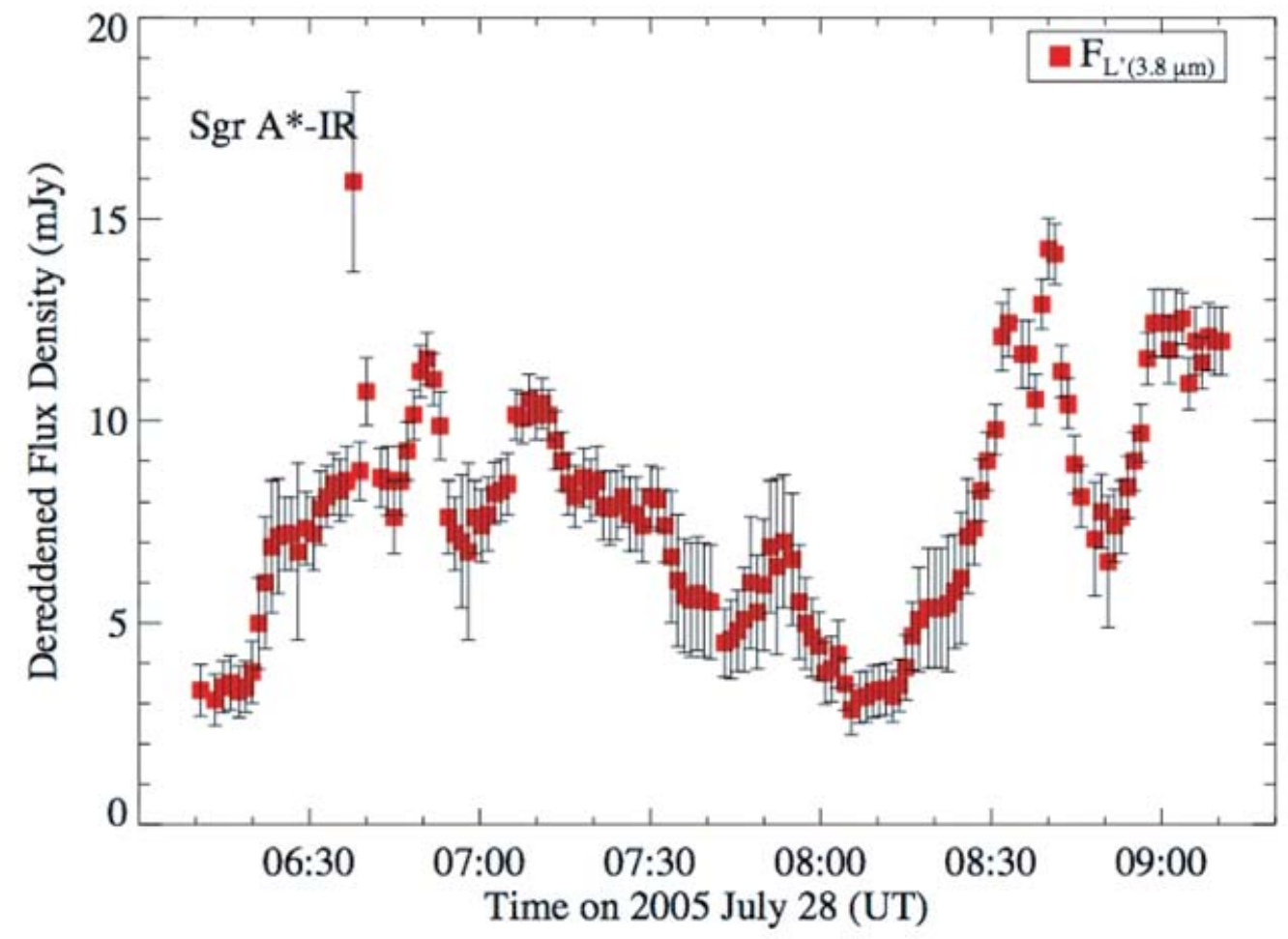

Figure 1. Light curve of Sgr A* at a wavelength of $3.8 \mu \mathrm{m}$ on 28 July 2005. This observation with the NIRC2 camera on the Keck II telescope was simultaneous with an X-ray observation with the Chandra X-ray Observatory, but no X-ray activity was detected above level of the extended quiescent emission.

Variability on few-hour time scales was investigated in depth by Mauerhan et al. (2005), who observed fluctuations of only $20 \div 40 \%$ during an 8 -day observing period. They carried out a red noise analysis of the variations, and concluded that there was excess power on a time scale of $2.5 \mathrm{hr}$.

Variations on hourly time scales have also been seen at submillimeter wavelengths. Marrone et al. (2006ab; see also Eckart et al. 2006) report observations of Sgr A* with the Submillimeter Array, in which they see hour-time-scale variability on the order of $10 \div 20 \%$ in total intensity at 230 and $345 \mathrm{GHz}$. The polarized sub-mm intensity, however, shows a far greater variation, in both position angle and percent polarization. While we do not discuss the implications of the polarization variations here, we note that they open up an exciting new dimension of investigation; the polarization variability, for example, may arise as a result of aspect changes associated with rotation in the inner disk.

\section{Infrared variability}

Sgr A* was found to be a variable infrared source at the same time that it was first convincingly detected independently by Genzel et al. (2003) at 1.65 and $2.16 \mu \mathrm{m}$ and Ghez et al. (2004) at $3.8 \mu \mathrm{m}$. These studies reported so-called "flares" having $40 \div 80$ minute durations, and showing substructure on smaller time scales, including a possible 17-minute periodicity (Genzel et al. 2003). Since then, a number of observations, all employing adaptive optics, have shown many further examples of near-IR events in Sgr A* 


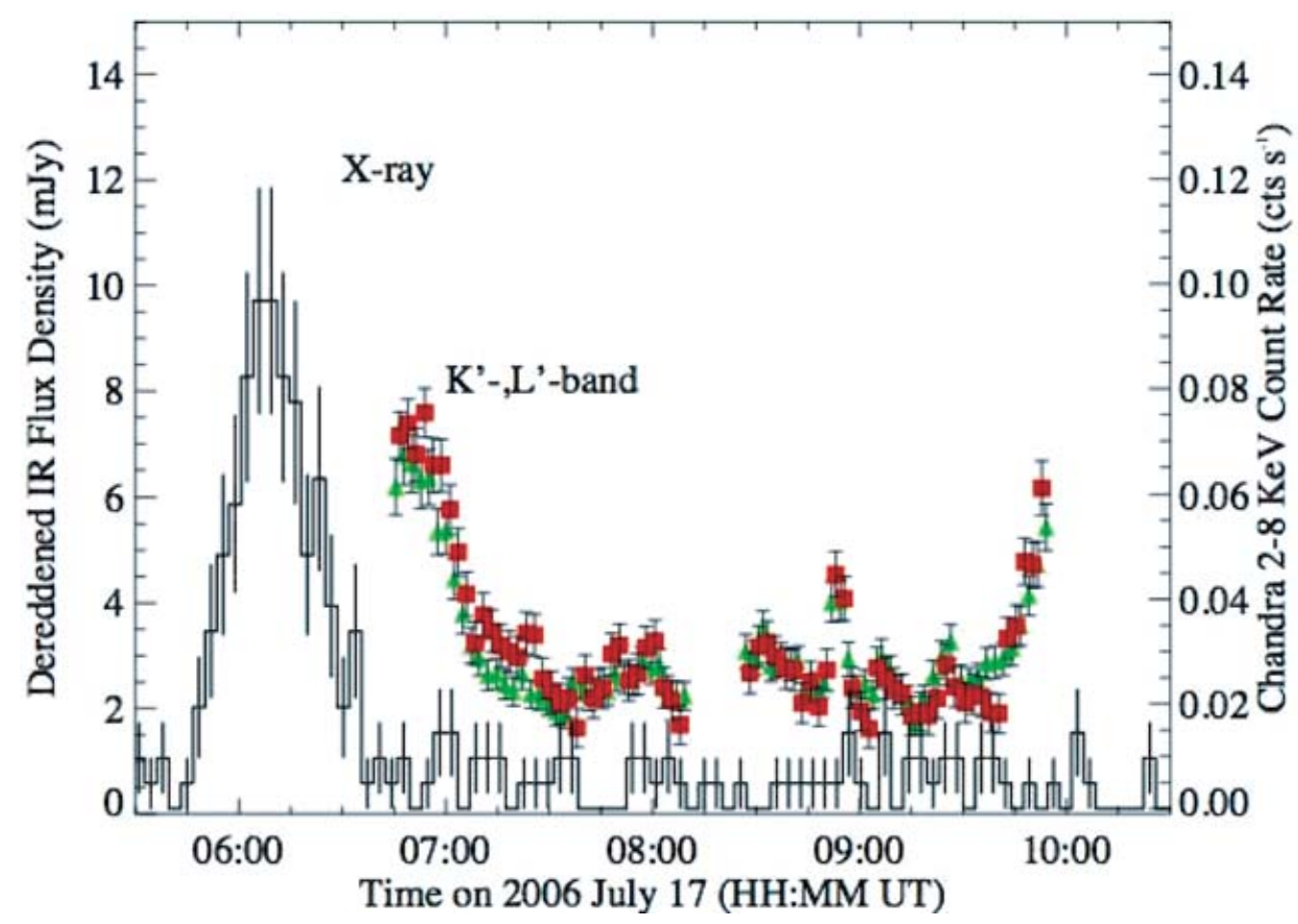

Figure 2. Light curves at $2.1 \mu \mathrm{m}$ (triangles), and $3.8 \mu \mathrm{m}$ (squares) measured alternately in quick succession on 17 July 2006; from Hornstein et al. (2007). When corrected for unresolved background starlight, the ratios of these fluxes are consistent with being constant. At bottom is the simultaneously obtained Chandra light curve, showing a substantial X-ray flare occurring just prior to the onset of the IR observations.

(Eckart et al. 2004, 2006, and in this volume; Hornstein et al. 2006; 2007). In the course of our Keck observations on several occasions over the past 3 years, we have not confirmed any quasi-periodicity on time scales comparable to 17 minutes, so the appearance of such quasi-periodicities in the total intensity does not appear to be commonplace.

The concept of a "flare" implies a temporary rise of the emission above some quiescent level, but it is not clear that a steady-state quiescent level exists in the near-IR. Indeed, the variability of Sgr $\mathrm{A}^{*}$ appears at $3.8 \mu \mathrm{m}$ to be continuous (figure 1), perhaps characterizable as a red noise light curve. At $2.1 \mu \mathrm{m}$, the flux density has been seen to range from $<2$ to $27 \mathrm{mJy}$ (Hornstein et al. 2007). However, it appears likely that, at both wavelengths, there is excess power on time scales of a few hours, such that the broad peaks manifest themselves as flares. In recognition of the continuous variability, Eckart et al. $(2004,2006)$ refer the intensity of their near-IR flares to an inconstant "interim quiescent" level. As with submillimeter emission, the polarized near-IR emission is more variable than the total intensity (Eckart et al., this volume).

\section{X-ray variability}

Since the first discovery of an X-ray flare from Sgr A* by Baganoff et al. (2001), many other X-ray flares having a broad range of intensities have been found with both Chandra and XMM (Baganoff 2005; Belanger et al. 2005, and references therein). The flares last from $30 \div 170$ minutes, and occur about once per day, with intensities so far ranging up 
to $\sim 150$ times the quiescent X-ray level. The quiescent level in X-rays is owed to partially resolved, perhaps thermal emission from the accretion flow, so is unrelated to the variable emission, which arises from within $10 \div 20 R_{s}$. The fact that X-ray peaks are significantly rarer than the $5 \div 12$ near-IR peaks per day has been attributed to a highly variable high-energy tail on the emitting electron distribution, whether the emission process be inverse Compton or synchrotron (Hornstein et al. 2007, and references therein). Several instances of simultaneous near-IR and X-ray peaks have been noted (Eckart et al. 2004, 2006; Yusef-Zadeh et al. 2006b), and it appears that all X-ray flares may be accompanied by a near-IR peak. It is possible that all of the near-IR peaks arising near Sgr A* are accompanied by X-ray peaks, but that only a few of these are sufficiently strong to rise above the quiescent level.

\section{The near-infrared spectrum}

Using the NIRC2 camera on the Keck II Telescope on several occasions in 2005 and 2006, we carried out the first time-series measurements, from 1.6 to $4.7 \mu \mathrm{m}$, of the broadband near-IR colors of Sgr A*. During our observations, the flux densities of Sgr A* varied widely, showing multiple peaks in the light curves (figure 2 shows the best-studied example). In spite of the intensity variations, however, the spectral slope of Sgr A* was remarkably constant: $\alpha=-0.6 \pm 0.2\left(F_{\nu} \propto \nu^{\alpha}\right)$. The same slope (within the uncertainties) was found for all observations at all wavelengths observed, regardless of the presence or absence of a nearby X-ray flare, and on all observing dates. The constancy of the IR spectral index raises the question of whether one can connect the infrared and X-ray emission to the same electrons.

We hypothesize that the electron acceleration mechanism produces a strikingly reproducible energy spectrum for the electrons responsible for the near-IR emission, while it occasionally generates a tail of electrons above $1 \mathrm{GeV}$ that, when sufficiently populated, gives rise to X-ray outbursts that accompany some of the near-IR peaks. If the electrons are accelerated in a turbulent medium, perhaps induced by a disk instability, then the high-energy cutoff in the electron energy spectrum might be linked to the presumably variable outer scale length of the turbulence.

\section{Acknowledgements}

This research has been supported by NSF grant AST-0406816 to UCLA.

\section{References}

Baganoff, F. K. et al. 2001, Nature, 413, 45

Baganoff, F. K. 2005, KITP Conference: Paradoxes of Massive Black Holes: A Case Study in the Milky Way, http://online.kitp.ucsb.edu/online/galactic_c05/baganoff

Belanger, G. et al. 2005, ApJ, 635, 1095

Belanger, G., Terrier, R., de Jager, O. C., Goldwurm, A. \& Melia, F. 2006, in: R. Schödel, G. C. Bower, M. P. Muno, S. Nayakshin \& T. Ott (eds.), J. Phys. Conf. Ser., 54, 420

Brown, R. L. \& Lo, K. Y. 1982, ApJ, 253, 225

Eckart, A. et al. 2004, A\&A, 427, 1

Eckart, A. et al. 2006, A\&A, 450, 535

Genzel, R. et al. 2003, Nature, 425, 943

Ghez, A. M. et al. 2004, ApJL, 601, L159

Herrnstein, R. M., Zhao, J.-H., Bower, G. C. \& Goss, W. M. 2004, AJ, 127, 3399

Hornstein, S. et al. 2004, in: R. Schödel, G. C. Bower, M. P. Muno, S. Nayakshin \& T. Ott (eds.), J. Phys. Conf. Ser., 54, 399 
Hornstein, S. et al. 2007, ApJ, submitted

Macquart, J.-P. \& Bower, G. C. 2006, ApJ, 641, 302

Marrone, D. P., Moran, J. M., Zhao, J.-H. \& Rao, R. 2006a, ApJ, 640, 308

Marrone, D. P., Moran, J. M., Zhao, J.-H. \& Rao, R. 2006b, in: R. Schödel, G. C. Bower, M. P. Muno, S. Nayakshin \& T. Ott (eds.), J. Phys. Conf. Ser., 54, 354

Mauerhan, J. C., Morris, M., Walter, F. \& Baganoff, F K. 2005, ApJL, 623, L25

Miyazaki, A., Tsutsumi, T. \& Tsuboi, M. 2004, ApJL, 611, L97

Miyazaki, A., Shen, Z.-Q., Miyoshi, M., Tsuboi, M. \& Tsutsumi, T. 2006, in: R. Schödel, G. C. Bower, M. P. Muno, S. Nayakshin \& T. Ott (eds.), J. Phys. Conf. Ser., 54, 363

Yusef-Zadeh, F., Roberts, D., Wardle, M., Heinke, C. O. \& Bower, G. C. 2006a, ApJ, 650, 189

Yusef-Zadeh, F. et al. 2006b, ApJ, 644, 198

Zhao, J.-H., Ekers, R. D., Goss, W. M., Lo, K. Y. \& Narayan, R. 1989, in: M. Morris (ed.), The Center of the Galaxy (Kluwer: Dordrecht), p. 535

Zhao, J.-H., Goss, W. M., Lo, K. Y. \& Ekers, R. D. 1992, in: A. Filippenko (ed.), Relationships Between Active Galactic Nuclei and Starburst Galaxies, ASP Conference Series, vol. 31, p. 295

Felix Mirabel: There are time-lags between $22 \mathrm{GHz}$ and $43 \mathrm{GHz}, \mathrm{K}$-band and X-rays etc., roughly up to order of one hour. This may suggest an adiabatic expansion. How would you accommodate this fact in a rotating medium of the disc?

MARK MORRIS: A sudden energy release can be responsible for sudden rise of intensity and can be accompanied with the expansion. This is consistent with recent results. However, it is baffling that the near-infrared spectral indices do not change through these strong variations of the intensity. 


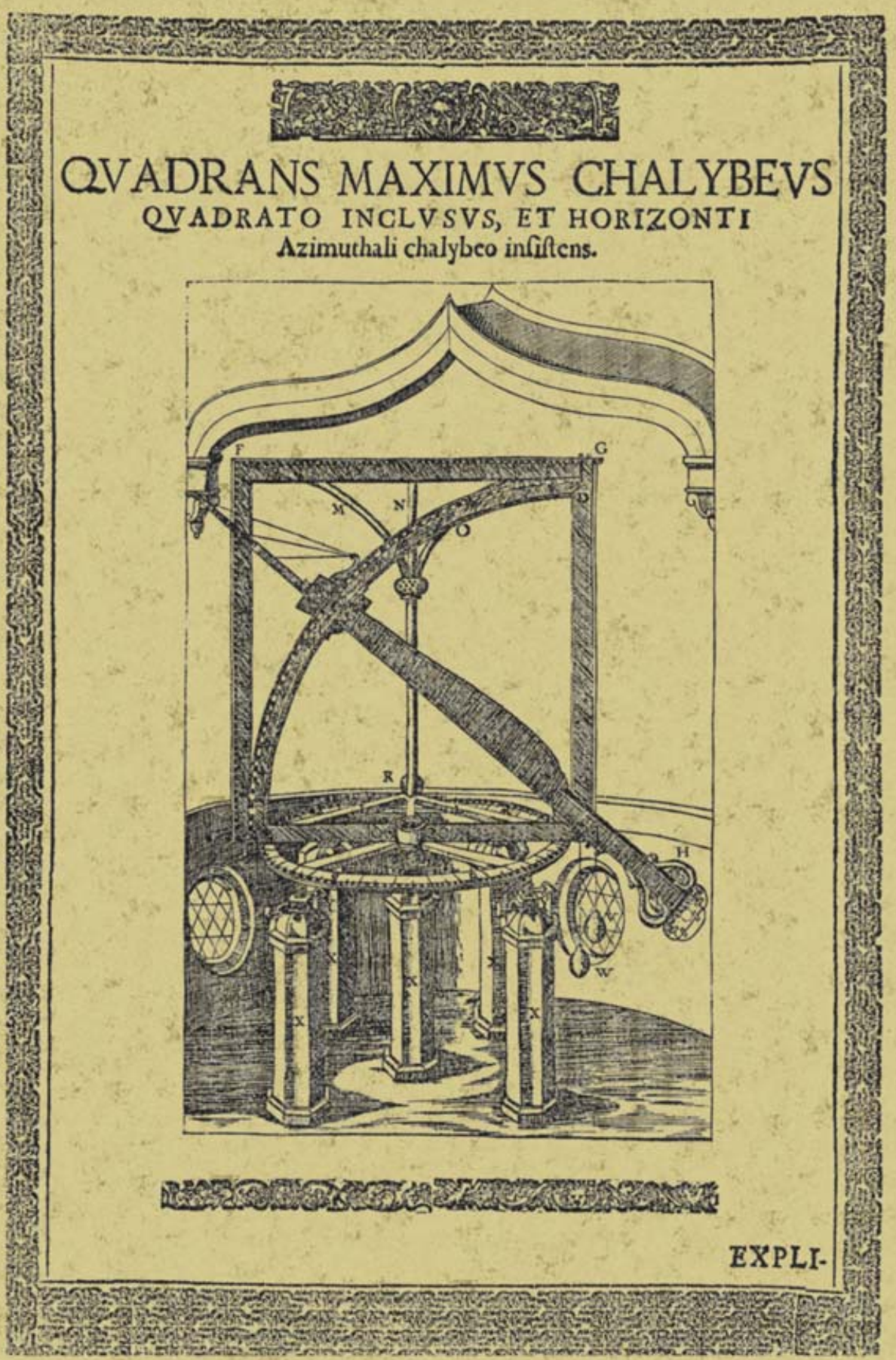

